\title{
The effect of tumor suppressor Pdcd4 knockdown on the malignancy of mouse melanoma cells
}

\section{Eriko Kinoshita ${ }^{1}$, Noriko Yoshikawa ${ }^{1}$, Mayuko Hatai ${ }^{1}$, Satomi Kagota ${ }^{2}$, Kazumasa Shinozuka ${ }^{2}$ Kazuki Nakamura ${ }^{1}$}

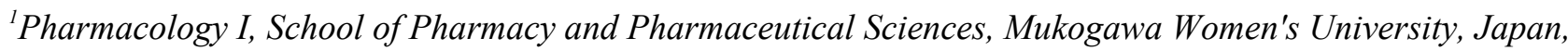
${ }^{2}$ Pharmacology II, School of Pharmacy and Pharmaceutical Sciences, Mukogawa Women's University, Japan

Purpose: Programmed cell death $4(\operatorname{Pdcd} 4)$ is a novel tumor suppressor gene which is known to act as a negative regulator of protein translation and malignant transformation. However, the direct targets of Pdcd4 remain unclear. We previously demonstrated that the presence of a negative correlation between the metastatic ability of tumor cells and expression levels of Pdcd4 in cells. In order to elucidate the mechanism of the inhibitory action of Pdcd4 on tumor malignancy, we conducted the knockdown of Pdcd4 in cells.

Methods: We used two mouse cell lines, B16-F0 and C57BL/6J-emb in this study. B16-F0 cells are low-metastatic melanoma cells and C57BL/6J-emb cells are normal fibroblast cells. We established the Pdcd4 knockdown cells using siRNA systems. The expression levels of Pdcd4 and the amount of secreted matrix metalloproteinase-2 (MMP-2) from cells were determined by Western blotting. The invasive ability of cells were measured by the chemoinvasion assay and the migration ability of cells were measured by the migration assay. We measured the survival time of syngeneic $\mathrm{C} 57 \mathrm{BL} / 6 \mathrm{Cr}$ mice intravenously inoculated with the Pdcd4 knockdown B16-F0 cells and compared with the mice injected the control cells.

Results: The Pdcd4 protein level in both B16-F0 cells and C57BL/6J-emb cells were significantly decreased after $24 \mathrm{hr}$ exposure to siPdcd4. Pdcd4 knockdown B16-F0 cells significantly increased their invasiveness and migration compared with the control cells. Similarly, Pdcd4 knockdown C57BL/6J-emb cells increased migration. Furthermore, the amount of secreted MMP-2 from Pdcd4 knockdown B16-F0 cells were significantly higher than the control cells. In addition, mice inoculated with Pdcd4 knockdown B16-F0 cells showed the tendency to reduce survival time compared with the control cells.

Summary/Conclusion: These results suggest that $\mathrm{Pdcd} 4$ suppresses the metastatic ability of tumor cells by controlling the invasive and migratory abilities of cells. 